\title{
QCD-like Theories at Finite Baryon and Isospin Density
}

\author{
K. SplittorfF ${ }^{1}$, D.T.Son ${ }^{2,4}$, and M.A. Stephanov ${ }^{3,4}$ \\ 1 The Niels Bohr Institute, Blegdamsvej 17, DK-2100 Copenhagen Ø, Denmark \\ 2 Physics Department, Columbia University, New York, NY 10027, USA \\ ${ }^{3}$ Department of Physics, University of Illinois, Chicago, IL 60607-7059, USA \\ ${ }^{4}$ RIKEN-BNL Research Center, Brookhaven National Laboratory, Upton, NY 11973, USA
}

September 10, 2018

\begin{abstract}
We use 2-color QCD as a model to study the effects of simultaneous presence of chemical potentials for isospin charge, $\mu_{I}$, and for baryon number, $\mu_{B}$. We determine the phase diagrams for 2 and 4 flavor theories using the method of effective chiral Lagrangians at low densities and weak coupling perturbation theory at high densities. We determine the values of various condensates and densities as well as the spectrum of excitations as functions of $\mu_{I}$ and $\mu_{B}$. A similar analysis of QCD with quarks in the adjoint representation is also presented. Our results can be of relevance for lattice simulations of these theories. We predict a phase of inhomogeneous condensation (FuldeFerrel-Larkin-Ovchinnikov phase) in the 2 colour 2 flavor theory, while we do not expect it the 4 flavor case or in other realizations of QCD with a positive measure.
\end{abstract}




\section{Introduction}

QCD at non-zero chemical potential has been a subject of numerous studies recently. The phenomenon of quark-quark pairing and color-superconductivity [1] has received renewed attention in view of the recent observations [2] that the superconducting gaps can be large and of possible relevance in astrophysics and heavy ion collisions (see [3] for a review). The most interesting finite density phenomena (phase transitions, in particular) occur in the non-perturbative regime, thus inviting lattice calculation methods. However, the lack of positivity of the Euclidean path integral prevents straightforward application of lattice Monte Carlo techniques. QCD at finite density thus remains a theoretical challenge.

This is one of the reasons that certain QCD-like theories with positive Euclidean path integral measure have also attracted attention. Examples of such theories are 2-color QCD, QCD with adjoint quarks, or 2-flavor QCD with isospin chemical potential 4, 6, 6, 7, 8]. In these theories, for each quark with a chemical potential $\mu$ there is another (conjugate) quark with exactly the same properties, except for the opposite sign of $\mu$. The quenched approximation, common in lattice studies, is an approximation to such kind of theories, rather than to QCD at finite baryon density with all quarks having equal chemical potentials [9].

In reality, dense baryon or quark matter, such as that arising in the interior of neutron stars or heavy ion collisions, is characterized by different, not equal and not opposite, chemical potentials for different flavors of quarks. One can describe such situations by the simultaneous presence of chemical potentials for baryon charge, $\mu_{B}$, and isospin $\mu_{I}$. The physics of this has been discussed [10] in the context of neutron stars $\left(\mu_{B} \gg \mu_{I}\right)$ and QCD at large density of isospin and small baryon density $\left(\mu_{I} \gg \mu_{B}\right)[8]$. The most interesting result is the appearance of a phase where ground state breaks translational and rotational symmetry. This phase is similar to the so-called FFLO (Fulde-FerrelLarkin-Ovchinnikov) phase of a BCS superconductor [11].

In this paper we investigate the behavior of QCD-like theories, in particular 2-color QCD under the influence of both $\mu_{I}$ and $\mu_{B}$. We shall use controllable analytical methods in two regimes of these theories: a chiral effective Lagrangian, based on the symmetries, in the low density regime, and weak coupling perturbation theory in the high density regime. We shall determine the phase diagram in the $\mu_{B}, \mu_{I}$ plane, various condensates, and lowest lying excitations.

Our goal is to gain an understanding of the physics of the interplay between $\mu_{B}$ and $\mu_{I}$. The theories we study (except $N_{c}=N_{f}=2$ ) have the advantage of having positive Euclidean path integral measure, and can, therefore, be studied on the lattice. Our results can be used as benchmarks for numerical lattice studies.

The presentation of our results is organized as follows. We start with two-colour QCD. In section 2 we give the low energy effective Lagrangian upon which the sequel is based. A qualitative argument for the structure of the phase diagram is then given in 3 . In section 0 we analyse the properties of the vacuum. As a result we find the classical values for the condensates and densities. Then in section 5 we study the expansion about the minimum in order to determine the masses of the low energy

excitations. The extension of this theory to $N_{f}=4$ with two up and two down flavours is discussed in section 6. The discussion of asymptotically high chemical potentials and the FFLO-phase is in section 7. Finally, in the last section we summarize and comment on the relevance of our results for real QCD. The presentation of QCD with quarks in the adjoint representation is placed in the appendix. 


\section{The Effective Theory at Finite $\mu_{B}$ and $\mu_{I}$}

Two colour QCD at zero chemical potential is invariant under $\mathrm{SU}\left(2 N_{f}\right)$ rotations in the chiral limit, see e.g [12]. This enhanced symmetry (as compared to the $\mathrm{SU}\left(N_{f}\right) \times \mathrm{SU}\left(N_{f}\right) \times \mathrm{U}(1)$ of three colour QCD) is manifest in the Lagrangian if we choose to represent this in a basis of quarks $\psi$ and conjugate quarks $\tilde{\psi}\left[\right.$ 田]. For $N_{f}=2$ we use

$$
\Psi \equiv\left(\begin{array}{c}
u \\
d \\
\tilde{u} \\
\tilde{d}
\end{array}\right) \equiv\left(\begin{array}{c}
u_{L} \\
d_{L} \\
\sigma_{2} \tau_{2}\left(u_{R}\right)^{*} \\
\sigma_{2} \tau_{2}\left(d_{R}\right)^{*}
\end{array}\right)
$$

where the Pauli matrices $\tau_{2}$ and $\sigma_{2}$ act in colour and spin space respectively.

The enhanced symmetry manifests itself in the low energy effective theory through the manifold of the Goldstone modes associated with the spontaneous breaking of chiral symmetry. In our case $N_{f}=2$ and the Goldstone manifold is $\mathrm{SU}(4) / \mathrm{Sp}(4)$, corresponding to the condensation of $\Psi \Psi-$ SU(4) flavor sextet. The fields on this manifold can be represented by a $4 \times 4$ antisymmetric unitary matrix $\Sigma$ with $\operatorname{det} \Sigma=1$.

The effective Lagrangian for the field $\Sigma$ of Goldstone modes is determined by the symmetries inherited from the microscopic two-colour QCD Lagrangian. In the basis of SU(4) spinors (1) the mass matrix, baryon charge matrix, and the isospin (third component) charge matrix aref

$$
\mathcal{M} \equiv\left(\begin{array}{cccc}
0 & 0 & 1 & 0 \\
0 & 0 & 0 & 1 \\
-1 & 0 & 0 & 0 \\
0 & -1 & 0 & 0
\end{array}\right), B \equiv \frac{1}{2}\left(\begin{array}{cccc}
1 & 0 & 0 & 0 \\
0 & 1 & 0 & 0 \\
0 & 0 & -1 & 0 \\
0 & 0 & 0 & -1
\end{array}\right), I \equiv \frac{1}{2}\left(\begin{array}{cccc}
1 & 0 & 0 & 0 \\
0 & -1 & 0 & 0 \\
0 & 0 & -1 & 0 \\
0 & 0 & 0 & 1
\end{array}\right)
$$

and the resulting effective Lagrangian is

$$
\begin{aligned}
\mathcal{L}_{\text {eff }}(\Sigma)= & \frac{F^{2}}{2} \operatorname{Tr} \partial_{\nu} \Sigma \partial_{\nu} \Sigma^{\dagger}+2 F^{2} \operatorname{Tr}\left(\left(\mu_{B} B+\mu_{I} I\right) \Sigma^{\dagger} \partial_{0} \Sigma\right) \\
& -F^{2} \operatorname{Tr}\left(\Sigma\left(\mu_{B} B+\mu_{I} I\right) \Sigma^{\dagger}\left(\mu_{B} B+\mu_{I} I\right)+\left(\mu_{B} B+\mu_{I} I\right)^{2}\right)-F^{2} m_{\pi}^{2} \operatorname{Re} \operatorname{Tr}(\mathcal{M} \Sigma)
\end{aligned}
$$

The $\mu$-dependent terms in the effective Lagrangian appear through the covariant extension of the derivative:

$$
\begin{aligned}
\partial_{0} \Sigma & \rightarrow \partial_{0} \Sigma-\left[\left(\mu_{B} B+\mu_{I} I\right) \Sigma+\Sigma\left(\mu_{B} B+\mu_{I} I\right)^{T}\right] \\
\partial_{0} \Sigma^{\dagger} & \rightarrow \partial_{0} \Sigma+\left[\left(\mu_{B} B+\mu_{I} I\right) \Sigma+\Sigma\left(\mu_{B} B+\mu_{I} I\right)^{T}\right]^{\dagger}
\end{aligned}
$$

required by an extended local gauge symmetry [ [4]. Therefore, to this order in chiral perturbation theory, the Lagrangian at finite $\mu$ does not require any extra phenomenological parameters beyond the pion decay constant, $F$, and the chiral condensate in the chiral limit, $G$. This fact gives predictive power to chiral perturbation theory at finite $\mu$. The chiral condensate can be traded for the vacuum pion mass using the Gell-Mann-Oaks-Renner relation $\left(m=m_{u}=m_{d}\right.$ is the quark mass):

$$
F^{2} m_{\pi}^{2}=m G
$$

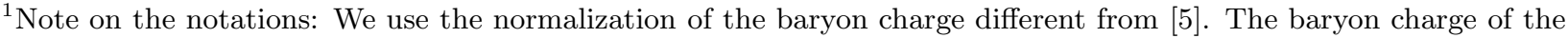
quark is not 1 , as in [5], but $1 / 2$, which comes from $1 / N_{c}$, so that the baryon (diquark in $N_{c}=2$ ) has baryon charge 1 . For simplicity, we omit subscript 3 from $I_{3}$.
} 
In using the effective Lagrangian constructed above we must of course assume that chiral symmetry for $N_{c}=N_{f}=2$ QCD is spontaneously broken. Since we have regarded the hadronic modes as heavy the theory is expected to be valid only up to the mass of the lightest non-goldstone hadron. However, as we shall show the phase diagram is very rich in the effective range of the theory.

\section{The phase diagram expected from the $\mu_{I}=0$ spectrum}

One can understand main features of the phase diagram in the $\mu_{B} \mu_{I}$ plane by considering the lowenergy spectrum of the theory at finite $\mu_{B}$ determined in [5]. In the vacuum, $\mu_{B}=\mu_{I}=0$, the spectrum consists of a degenerate 5-plet: 3 pions $\pi_{0}, \pi^{ \pm}(B=0, I=0, \pm 1)$, a baryon and an antibaryon $q, q^{*}$ (diquark and antidiquark with $B= \pm 1$ and $I=0$ ).

On the horizontal axis, $\mu_{I}=0$, as a function of $\mu_{B}$, there is a phase transition corresponding to the condensation of diquarks. This happens at $\mu_{B}$ equal to the diquark mass divided by its baryon charge, i.e., at $\mu_{B}=m_{\pi}$. Similarly, at $\mu_{B}=0$ there should be a transition corresponding to the condensation of pions. This happens when $\mu_{I}$ is equal to the pion mass divided by its isospin charge, i.e., at $\mu_{I}=m_{\pi}$. This can also be concluded from the fact that there is a simple discrete symmetry: $d \leftrightarrow \tilde{d}$ accompanied by $\mu_{B} \leftrightarrow \mu_{I}$. Therefore, the $\mu_{B} \mu_{I}$ phase diagram must be symmetric under reflection about $\mu_{B}=\mu_{I}$ line.

As a function of $\mu_{B}$ the mass of the pion is constant as long as $\mu_{B}<m_{\pi}$, which means the transition at $\mu_{I}=m_{\pi}$ happens at all such $\mu_{B}$. Related to this horizontal line of phase transitions by a reflection against the diagonal, there is a vertical line at $\mu_{B}=m_{\pi}$, the line where diquarks condense. After the two lines meet they must merge into a single $\mu_{B}=\mu_{I}$ line. This is because of the reflection symmetry and the fact that the mass of the pion in the diquark condensation phase $\mu_{B}>m_{\pi}$ is equal to $\mu_{B}$ according to [5]. Two different condensation modes (pion vs diquark) compete at $\mu_{B}=\mu_{I}$, thus the transition along the $\mu_{B}=\mu_{I}$ line is of first order.

\section{Minimum of the effective Lagrangian}

In order to derive the phase diagram we study the minimum of the classical theory. That is: we seek to minimize the static part of the effective Lagrangian

$$
\mathcal{L}_{\text {eff }}(\bar{\Sigma})=-F^{2} \operatorname{Tr}\left(\bar{\Sigma}\left(\mu_{B} B+\mu_{I} I\right) \bar{\Sigma}^{\dagger}\left(\mu_{B} B+\mu_{I} I\right)+\left(\mu_{B} B+\mu_{I} I\right)^{2}\right)-F^{2} m_{\pi}^{2} \operatorname{Re} \operatorname{Tr}(\mathcal{M} \bar{\Sigma})
$$

of the Lagrangian (3)). Our ansatz for the minimum is

$$
\bar{\Sigma} \equiv \Sigma_{M} \cos \alpha+\left(\Sigma_{B} \cos \eta+\Sigma_{I} \sin \eta\right) \sin \alpha
$$

where $\alpha$ and $\eta$ are variational parameters, $\Sigma_{M} \equiv-\mathcal{M}$, while $\Sigma_{B}$ and $\Sigma_{I}$ are given by

$$
\Sigma_{B}=\left(\begin{array}{cccc}
0 & -i & 0 & 0 \\
i & 0 & 0 & 0 \\
0 & 0 & 0 & -i \\
0 & 0 & i & 0
\end{array}\right) \quad \text { and } \quad \Sigma_{I}=\left(\begin{array}{cccc}
0 & 0 & 0 & i \\
0 & 0 & i & 0 \\
0 & -i & 0 & 0 \\
-i & 0 & 0 & 0
\end{array}\right)
$$

The ansatz is based on the following observations: three terms compete for the alignment of the condensate: $m \mathcal{M}, \mu_{B} B$, and $\mu_{I} I$. Each of them is independently minimized by $\Sigma_{M}, \Sigma_{B}$, and $\Sigma_{I}$ respectively. 
Inserting the ansatz and using the (anti)commutation relations between the matrices involved we reduce the static Lagrangian to

$$
\mathcal{L}_{\text {eff }}(\bar{\Sigma})=-F^{2}\left(-\left(\mu_{B}^{2}+\mu_{I}^{2}\right) \cos ^{2} \alpha+\left(\mu_{B}^{2}-\mu_{I}^{2}\right) \cos (2 \eta) \sin ^{2} \alpha+\mu_{B}^{2}+\mu_{I}^{2}+4 m_{\pi}^{2} \cos \alpha\right) .
$$

Extremizing with respect to $\eta$ we find (for $\alpha \neq 0$ ):

$$
\begin{array}{cll}
\eta=0 & \text { if } & \mu_{I}<\mu_{B} \\
\eta=\frac{\pi}{2} & \text { if } & \mu_{I}>\mu_{B} .
\end{array}
$$

Note that for $\mu_{B}^{2}=\mu_{I}^{2}$ there is degeneracy in the $\eta$-direction. This is a manifestation of the $\mathrm{SU}(2)$ rotational symmetry (at $m=0$ ) between the flavor which has $\mu=0$ and its own conjugate field (i.e., between $d$ and $\tilde{d}$ in the first quadrant of the $\mu_{I}, \mu_{B}$-plane). Extremizing with respect to the $\alpha$-direction we find:

$$
\begin{array}{rll}
\alpha=0 & \text { if } & y>1 \\
\cos \alpha=y & \text { if } & y<1
\end{array}
$$

where

$$
\begin{aligned}
& y=\frac{m_{\pi}^{2}}{\mu_{B}^{2}} \quad \text { if } \quad \mu_{I}<\mu_{B} \\
& y=\frac{m_{\pi}^{2}}{\mu_{I}^{2}} \quad \text { if } \quad \mu_{I}>\mu_{B} .
\end{aligned}
$$

The ansatz (8) is indeed a minimum of the classical theory as we shall prove in the next section.

Since the vacuum energy is given by the value of the Lagrangian at the minimum we can draw the following conclusions

$$
\begin{aligned}
\langle\bar{\psi} \psi\rangle & =-\frac{\partial \mathcal{L}_{\text {eff }}(\bar{\Sigma})}{\partial m}=4 G \cos \alpha & \\
n_{B} & =-\frac{\partial \mathcal{L}_{\text {eff }}(\bar{\Sigma})}{\partial \mu_{B}}=2 F^{2} \mu_{B}(1+\cos (2 \eta)) \sin ^{2} \alpha & n_{I}=-\frac{\partial \mathcal{L}_{\text {eff }}(\bar{\Sigma})}{\partial \mu_{I}}=2 F^{2} \mu_{I}(1-\cos (2 \eta)) \sin ^{2} \alpha \\
\langle\psi \psi\rangle & =-\frac{\partial \mathcal{L}_{\text {eff }}(\bar{\Sigma})}{\partial j_{B}}=4 G \sin \alpha \cos \eta & \langle\pi\rangle=-\frac{\partial \mathcal{L}_{\text {eff }}(\bar{\Sigma})}{\partial j_{I}}=4 G \sin \alpha \sin \eta .
\end{aligned}
$$

To obtain the diquark and pion condensates we have introduced a diquark source, $j_{B}$, and a pion source, $j_{I}$, in (3). The condensates are the derivatives of $\mathcal{L}_{\text {eff }}(\bar{\Sigma})$ with respect to the sources. The introduction of the sources is analogous to that in [5] and we refer to that paper for details. The phase diagram is shown in the left hand side of fig.1. It is in agreement with our qualitative argument given in section 3. In particular the densities are discontinuous across at $\mu_{B}=\mu_{I}>m_{\pi}$ indicating a first order phase transition. Note, however, that the vacuum energy, Eq. (10), is smooth across the $\mu_{B}=\mu_{I}$ line. The values of the condensates are illustrated in figures 1 and 2 .

\section{Expanding About the Minimum}

In order to determine the phase diagram we had to find the minimum of the effective Lagrangian (3) with respect to $\Sigma$. Having found this, we now determine the masses of low-energy excitations by expanding around the minimum $\bar{\Sigma}$. 

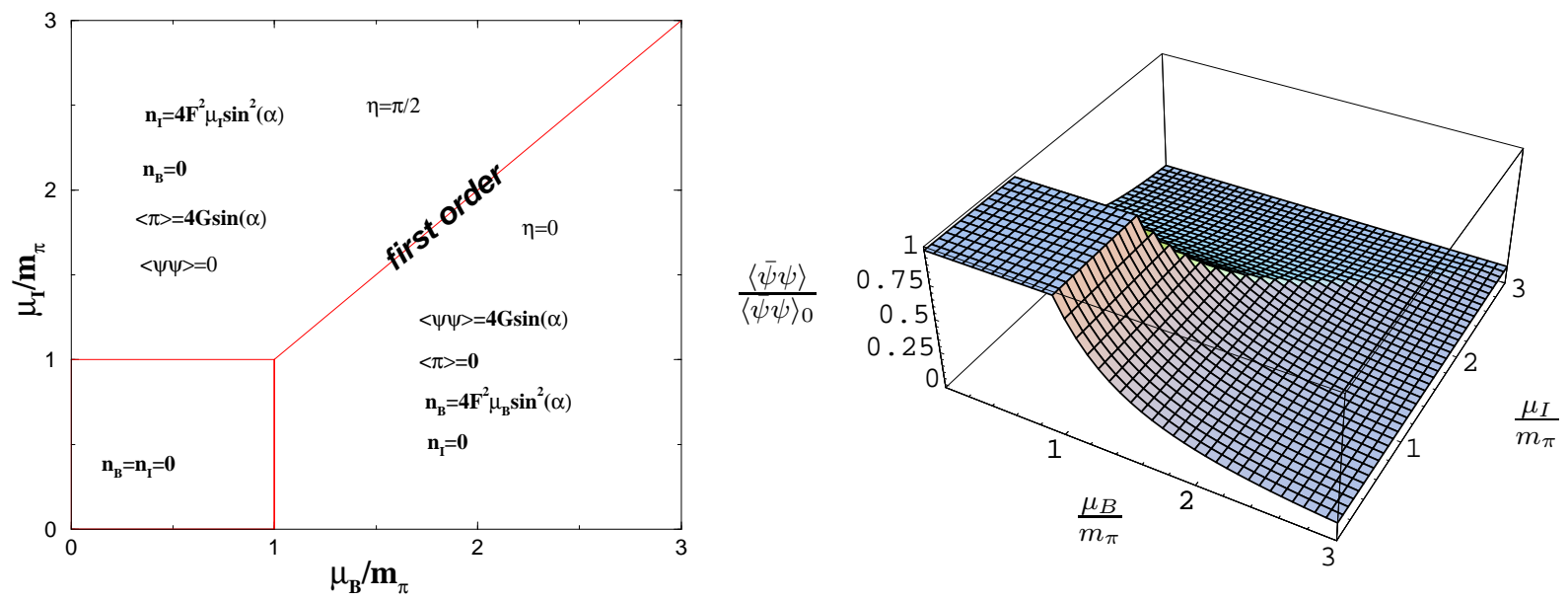

Figure 1: $\quad N_{c}=2, N_{f}=2\left(\beta_{\mathrm{D}}=1\right)$ : The figure on the left shows a schematic version of the phase diagram. To the right: The ratio of the chiral condensate to its value at $\alpha=0$ in the first quadrant of the $\left(\mu_{B}, \mu_{I}\right)$-plane.

\subsection{Parameterization of the field $\Sigma$}

A convenient way to parameterize the matrix $\Sigma$ in the representation of $\mathrm{SU}(4) / \mathrm{Sp}(4)$ is based on its $\mathrm{SU}(4)$ transformation properties [13]:

$$
\Sigma=U \bar{\Sigma} U^{\mathrm{T}}=U^{2} \bar{\Sigma}
$$

Here the second equality is imposed by the algebra of the group [13. The special unitary $4 \times 4$ matrix

$$
U=e^{i \frac{\Pi}{2 F}}
$$

contains the fluctuating fields $\Pi$. At $\mu=0$ the Lagrangian is minimized by $\Sigma=\Sigma_{M}$, aligned along the quark mass term $m \mathcal{M}$ :

$$
\Sigma_{M} \equiv\left(\begin{array}{cccc}
0 & 0 & -1 & 0 \\
0 & 0 & 0 & -1 \\
1 & 0 & 0 & 0 \\
0 & 1 & 0 & 0
\end{array}\right)
$$

With this choice the hermitian field $\Pi$ is restricted by the manifold to [13]

$$
\Pi \equiv \frac{1}{\sqrt{2}}\left(\begin{array}{cccc}
\tilde{p} & p & 0 & q \\
p^{*} & -\tilde{p} & -q & 0 \\
0 & -q^{*} & \tilde{p} & p^{*} \\
q^{*} & 0 & p & -\tilde{p}
\end{array}\right) .
$$

Where $\tilde{p}$ is real and $p$ and $q$ are complex numbers. The fields $\tilde{p}, p, p^{*}$ are neutral and charged pions and $q, q^{*}$ are diquark/antidiquarks. The $\bar{\Sigma}$ will change as a function of $\mu$ and we shall discuss the parameterization around a generic minimum below.

The purpose of carrying out an expansion of $\mathcal{L}_{\text {eff }}$ in the $\Pi$-fields about the minimum $\bar{\Sigma}$ is twofold. First, in order to justify that the ansatz (8) is a minimum, we need to prove that the linear terms in $\Pi$ vanish. Second, the expansion of $\mathcal{L}_{\text {eff }}$ allows us to determine the dispersion relations for the low energy excitations. 

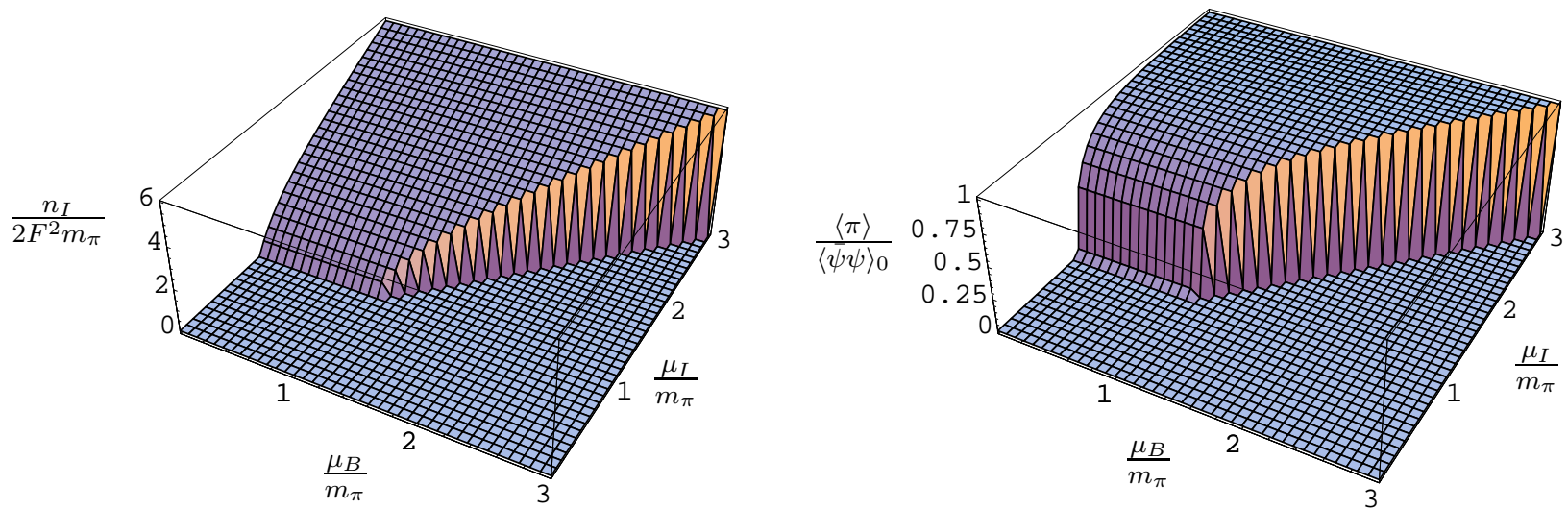

Figure 2: $\beta_{\mathrm{D}}=1, N_{f}=2$ : The leftmost plot displays the isospin charge density in the $\left(\mu_{B}, \mu_{I}\right)$-plane in units of $2 F^{2} m_{\pi}$. On the right hand side is shown the ratio of the pion condensate to the chiral condensate at $\alpha=0$. The first order phase transition is apparent.

\subsection{Linear Terms}

To expand $\mathcal{L}_{\text {eff }}$ around $\bar{\Sigma}$ defined in (8) we need to choose a parameterization of the $\Pi$-field which can be used for all values of $\alpha$ and $\eta$. For $\alpha=0$ we have already given a parameterization in (18). For a given pair $(\alpha>0, \eta)$ this parameterization of $\Sigma$ is no longer valid. We must expand about the rotated $\bar{\Sigma}$ according to (15). However, as it is useful not to change the parameterization (18) with $\alpha$ and $\eta$, we extract the rotation from $U$ and write it explicitly. To do this we first note that we can write the ansatz (8) as

$$
\bar{\Sigma}(\alpha, \eta)=e^{-\alpha\left(\Sigma_{B} \cos \eta+\Sigma_{I} \sin \eta\right) \Sigma_{M} \Sigma_{M}} \equiv V_{(\alpha, \eta)}^{2} \Sigma_{M} \equiv V_{(\alpha, \eta)} \Sigma_{M} V_{(\alpha, \eta)}^{\mathrm{T}} .
$$

Referring to (15) we see that

$$
\begin{aligned}
\Sigma(\alpha, \eta) & \equiv U(\alpha, \eta) \bar{\Sigma}(\alpha, \eta) U^{\mathrm{T}}(\alpha, \eta) \\
& =V_{(\alpha, \eta)} U(\alpha=0, \eta) V_{(\alpha, \eta)}^{\dagger} V_{(\alpha, \eta)} \Sigma_{M} V_{(\alpha, \eta)}^{\mathrm{T}} V_{(\alpha, \eta)}^{\dagger} U^{\mathrm{T}}(\alpha=0, \eta) V_{(\alpha, \eta)}^{\mathrm{T}} \\
& =V_{(\alpha, \eta)} \Sigma_{M} U^{2}(\alpha=0, \eta) V_{(\alpha, \eta)}^{T} .
\end{aligned}
$$

Inserting this in $\mathcal{L}_{\text {eff }}(3)$ we find that the form of the Lagrangian does not change provided that we substitute rotated values of $\mathcal{M}$ and $\mu_{B} B+\mu_{I} I$

$$
\begin{aligned}
V_{(\alpha, \eta)}^{T} \mathcal{M} V_{(\alpha, \eta)}= & -\Sigma_{M} \cos \alpha-\left(\Sigma_{B} \cos \eta+\Sigma_{I} \sin \eta\right) \sin \alpha \\
V_{(\alpha, \eta)}^{\dagger}\left(\mu_{B} B+\mu_{I} I\right) V_{(\alpha, \eta)}= & \left(\mu_{B} B \cos \alpha-\mu_{B} B \Sigma_{B} \Sigma_{M} \sin \alpha+\mu_{I} I\right) \cos \eta \\
& +\left(\mu_{I} I \cos \alpha-\mu_{I} I \Sigma_{I} \Sigma_{M} \sin \alpha+\mu_{B} B\right) \sin \eta .
\end{aligned}
$$

Using this and expanding $U=\exp (i \Pi / 2 F)$ to $2^{\text {nd }}$ order in $\Pi$ we find that the terms liner in $\Pi$ vanish. (The cancellation takes place due to the equations $(12,13)$ arising from the constraint $\partial_{\alpha} \mathcal{L}_{\text {st }}(\bar{\Sigma})=0$.) From this we conclude that the ansatz (8) for the minimum is at least a local extremum of the theory. As we now turn to focus on the terms quadratic in $\Pi$ we shall find that this local extremum is indeed a minimum. 


\subsection{Dispersion Relations}

The quadratic terms in $\Pi$ of the expansion around $\bar{\Sigma}$ determine the dispersion relations of the low energy excitations. For $\mu_{B}>\mu_{I}$ the terms in $\mathcal{L}_{\text {eff }}(\Sigma)$ at second order in $\Pi$ are

$$
\begin{aligned}
\mathcal{L}_{\text {eff }}^{(2)}(\Sigma)= & \frac{1}{2} \operatorname{Tr}\left\{\left(\partial_{\nu} \Pi-\mu_{I}[I, \Pi] \delta_{\nu 0}-\mu_{B} \cos \alpha[B, \Pi] \delta_{\nu 0}\right)^{2}\right. \\
& \left.+\mu_{B}^{2}\left[B \Sigma_{B} \Sigma_{M}, \Pi\right]^{2} \sin ^{2} \alpha+m_{\pi}^{2} \Pi^{2} \cos \alpha\right\}
\end{aligned}
$$

The corresponding expression for $\mu_{B}<\mu_{I}$ is obtained from the above by switching $B$ and $I$ symbols. Recall that we have kept the parameterization (18) at the cost of having rotated $\left(\mu_{B} B+\mu_{I} I\right)$ and $\mathcal{M}$, see (21). Using this parameterization of $\Pi$ we rewrite the above equation as

$$
\begin{aligned}
\mathcal{L}_{\mathrm{eff}}^{(2)}(\Sigma)= & \left(\partial_{\nu} \tilde{p}\right)^{2}+\left(\partial_{\nu} p-\mu_{I} \delta_{\nu 0} p\right)\left(\partial_{\nu} p^{*}+\mu_{I} \delta_{\nu 0} p^{*}\right)+\left(\partial_{\nu} q-\mu_{B} \delta_{\nu 0} q \cos \alpha\right)\left(\partial_{\nu} q^{*}+\mu_{B} \delta_{\nu 0} q^{*} \cos \alpha\right) \\
& +\frac{\mu_{B}^{2}}{4}\left(\left(q+q^{*}\right)^{2}+4\left(p p^{*}+\tilde{p}^{2}\right)\right) \sin ^{2} \alpha+m_{\pi}^{2}\left(\tilde{p}^{2}+p p^{*}+q q^{*}\right) \cos \alpha
\end{aligned}
$$

This allows us to read off the dispersion relations for each of the 5 modes.

In the diquark condensation phase $\alpha \neq 0$ the $q$ and $q^{*}$ fields mix. The dispersion relations are obtained by solving for $E=i p_{0}$ :

$$
\operatorname{det}\left(\begin{array}{cc}
\mu_{B}^{2} \sin ^{2}(\alpha) / 2 & -\left(E-\mu_{B} \cos \alpha\right)^{2}+\mathbf{p}^{2} \\
-\left(E+\mu_{B} \cos \alpha\right)^{2}+\mathbf{p}^{2} & +\mu_{B}^{2} \sin ^{2}(\alpha) / 2+m_{\pi}^{2} \cos \alpha \\
+\mu_{B}^{2} \sin ^{2}(\alpha) / 2+m_{\pi}^{2} \cos \alpha & \mu_{B}^{2} \sin ^{2}(\alpha) / 2
\end{array}\right)=0 \text {. }
$$

The mixing between $q$ and $q^{*}$ implies that two of the low energy excitations are linear combinations of diquark and anti-diquark modes. We denote them $\tilde{q}$ and $\tilde{q}^{*}$. As these diquark/anti-diquarks carry no isospin it is not surprising that the dispersion relations are the same as found in [5]. In the normal phase $(\alpha=0)$

$$
\begin{array}{rlrl}
q^{*} & E & =\sqrt{\mathbf{p}^{2}+m_{\pi}^{2}}+\mu_{B} \\
q & E & =\sqrt{\mathbf{p}^{2}+m_{\pi}^{2}}-\mu_{B} .
\end{array}
$$

while for $\alpha \neq 0$

$$
\begin{array}{ll}
\tilde{q}^{*} & E^{2}=\mathbf{p}^{2}+\mu_{B}^{2}\left(1+3 \cos ^{2} \alpha\right) / 2+\mu_{B} \sqrt{\mu_{B}^{2}\left(1+3 \cos ^{2} \alpha\right)^{2} / 4+4 \mathbf{p}^{2} \cos ^{2} \alpha} \\
\tilde{q} & E^{2}=\mathbf{p}^{2}+\mu_{B}^{2}\left(1+3 \cos ^{2} \alpha\right) / 2-\mu_{B} \sqrt{\mu_{B}^{2}\left(1+3 \cos ^{2} \alpha\right)^{2} / 4+4 \mathbf{p}^{2} \cos ^{2} \alpha} .
\end{array}
$$

Note that one mode is massless for $\mu_{B}>m_{\pi}$.

There is no mixing in the $\tilde{p}, p, p^{*}$ sector. This implies that we can interpret $\tilde{p}, p$, and $p^{*}$ as $\pi^{0}, \pi^{+}$ and $\pi^{-}$and directly read off the dispersion relations: For $\alpha=0$

$$
\begin{array}{ll}
\pi^{0} & E=\sqrt{\mathbf{p}^{2}+m_{\pi}^{2}} \\
\pi^{+} & E=\sqrt{\mathbf{p}^{2}+m_{\pi}^{2}}+\mu_{I} \\
\pi^{-} & E=\sqrt{\mathbf{p}^{2}+m_{\pi}^{2}}-\mu_{I}
\end{array}
$$



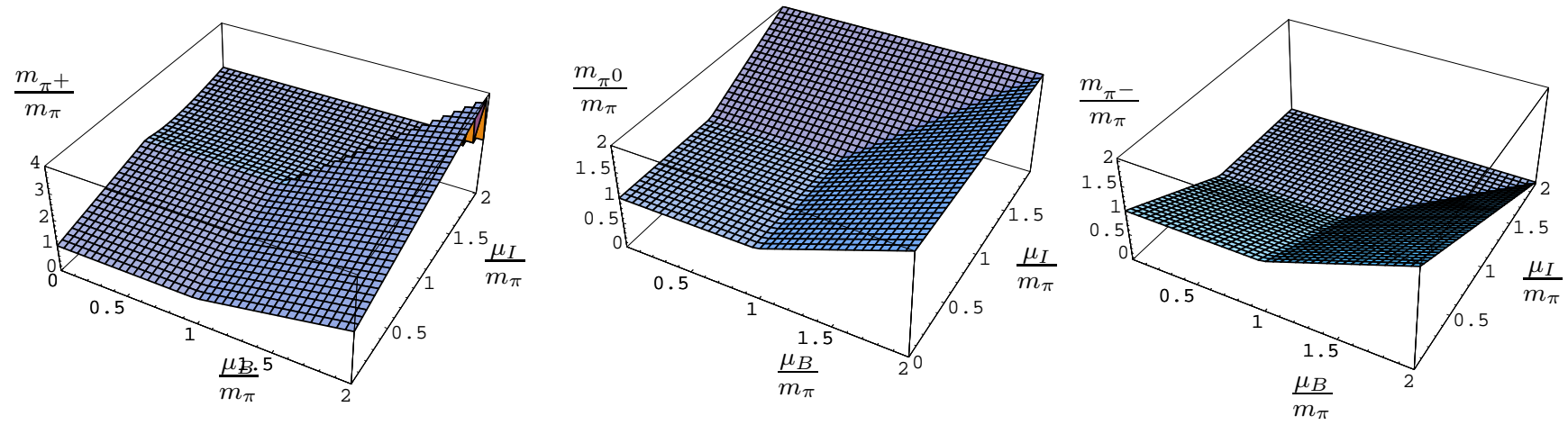

Figure 3: $\beta_{\mathrm{D}}=1, N_{f}=2$ : The ratio masses of the pion modes to $m_{\pi}$ as a function of $\mu_{B}$ and $\mu_{I}$. From the left $m_{\pi^{+}}, m_{\pi^{0}}$, and $m_{\pi^{-}}$. Note that the mass of the $\pi^{-}$excitation vanishes at $\mu_{B}=\mu_{I}>m_{\pi}$. The masses of the $\tilde{q}^{*}$ and the $\tilde{q}$ excitations are the mirrors in the $\mu_{B}=\mu_{I}$-plane of $m_{\pi}^{+}$and $m_{\pi}^{-}$ respectively.

while in the diquark condensation phase $(\alpha \neq 0)$

$$
\begin{array}{ll}
\pi^{0} & E=\sqrt{\mathbf{p}^{2}+\mu_{B}^{2}} \\
\pi^{+} & E=\sqrt{\mathbf{p}^{2}+\mu_{B}^{2}}+\mu_{I} \\
\pi^{-} & E=\sqrt{\mathbf{p}^{2}+\mu_{B}^{2}}-\mu_{I} .
\end{array}
$$

As one might expect we find that the $\pi^{+}$and $\pi^{-}$modes couple to $\mu_{I}$. For $\mu_{B}=\mu_{I}>m_{\pi}$ the $\pi^{-}$ becomes massless. For plots of the masses see figure 3 .

The dispersion relations for $\mu_{B}<\mu_{I}$ in the first quadrant are mirrors of the ones above. To obtain them one needs only to switch $\mu_{I} \leftrightarrow \mu_{B}$ in (25)-(28) and charged pions with diquarks/anti-diquarks: $p \leftrightarrow q$.

Finally, since the masses of the excitations are positive the value of $\mathcal{L}_{\text {eff }}(\bar{\Sigma})$ with $\bar{\Sigma}$ given in $(8)$ is not only an extremum as shown in the previous section, but is indeed a minimum. We assume that this minimum is global.

\section{Positivity and $N_{f}=4$ phase diagram}

Testing these results in lattice Monte Carlo simulations requires care. The fermion determinant, though always real in 2-color QCD, is not positive definite if both $\mu_{B}$ and $\mu_{I}$ are non-zero. This can be seen by factorizing the determinant into a product of the determinants for $u$ and $d$ quarks with chemical potentials $\mu_{u, d}=\mu_{B} \pm \mu_{I}$. Each of these determinants is real but not positive definite if $\mu_{u, d} \neq 0$. So, unless $\mu_{u}= \pm \mu_{d}$, their product is not positive definite. Doubling the number of quarks to save positivity will take us to the $N_{f}=4$ theory, with a significantly different phase diagram, which we shall now discuss. [2

One can understand the phase diagram in this $N_{f}=4$ theory (two up quarks $u_{1,2}$ and two down quarks $\left.d_{1,2}\right)$ qualitatively using the arguments of sect. 3. The most important difference is that there

\footnotetext{
${ }^{2}$ We assume that the chiral symmetry is still broken at $N_{f}=4$. The results are trivially extended to $2 N u$-quarks and $2 N d$-quarks if chiral symmetry is assumed to be spontaneously broken for that number of flavours. Likewise the results on adjoint QCD presented in the appendix may be extended to $N u$-quarks and $N d$-quarks.
} 


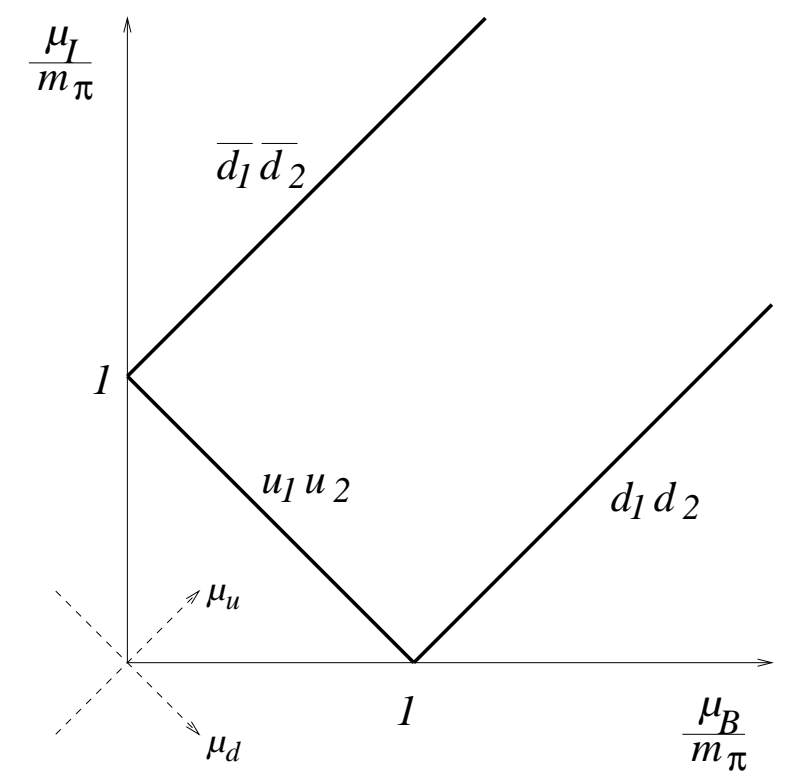

Figure 4: Phase diagram of the $N_{f}=42$-color QCD, determined by a qualitative argument of section 6. Solid lines are (second order) phase transitions where certain diquark/antidiquark condensates, indicated on the appropriate side of each line, appear. Subscripts refer to flavour. Dashed lines show the direction of $\mu_{u}$ and $\mu_{d}$ axes.

are now diquarks which carry isospin: there is an isospin triplet $\left(u_{1} u_{2}, d_{1} d_{2}, u_{1} d_{2}+d_{1} u_{2}\right)$ and 3 isosinglets. As $\mu_{B}$ increases, the masses of all 6 diquarks are decreasing as $m_{\pi}-\mu_{B}$ [5]. Among them is $u_{1} u_{2}$, which becomes the lightest particle with positive isospin. Therefore, for a given $\mu_{B}<m_{\pi}$, the isospin charge condensation occurs at $\mu_{I}=m_{\pi}-\mu_{B}$ (instead of the horizontal line $\mu_{I}=m_{\pi}$ as in $N_{f}=2$ ) - compare figs. 1 and 1 . At $\mu_{B}>m_{\pi}$ the diquarks (including the ones carrying isospin) are massless and the isospin is condensed already at $\mu_{I}=0$. By the $\mu_{B} \leftrightarrow \mu_{I}$ reflection symmetry, the baryon charge condensation for $\mu_{I}>m_{\pi}$ sets in at $\mu_{B}=0$. The region of the phase diagram to the right of (or above) the line $\mu_{I}=m_{\pi}-\mu_{B}$ contains a condensate of $u_{1} u_{2}$, which is favoured by both $\mu_{B}$ and $\mu_{I}$.

To complete the phase diagram in fig. (1 note that the line $\mu_{I}=m_{\pi}-\mu_{B}$ where $u_{1} u_{2}$ diquark condensation occurs continues also into the negative $\mu_{I}$ halfplane. Because the theory is invariant under $\mu_{I} \rightarrow-\mu_{I}, d \leftrightarrow u$ substitution, a line $m_{I}=\mu_{B}-m_{\pi}$ must exist in the positive $\mu_{B} \mu_{I}$ quadrangle (see fig.(1). Below this line the condensate of diquarks $d_{1} d_{2}$, carrying isospin -1 , appears. By $\mu_{B} \leftrightarrow \mu_{I}$, $d \leftrightarrow \bar{d}$ reflection we obtain another line, $\mu_{I}=\mu_{B}+m_{\pi}$ above which the condensate of $\bar{d}_{1} \bar{d}_{2}$ appears.

An easier way to understand the phase diagram of fig. 4 is to consider $u$ and $d$ quarks separately. Rotating by $45^{\circ}$ and using $\mu_{u}$ and $\mu_{d}$ axes one sees that the diagram is completely symmetric with respect to $u$ and $d$ quarks, with condensation of $u_{1} u_{2}$ and $d_{1} d_{2}$ diquarks or antidiquarks occurring independently once $\left|\mu_{u}\right|$ or $\left|\mu_{d}\right|$ exceed the vacuum mass $m_{\pi}$.

We have deferred the rigorous derivation of this phase diagram to future work. However, the phase diagram of another theory: 2- (or any-) color QCD with $N_{f}=2$ adjoint quarks (Dyson index $\beta_{\mathrm{D}}=4$ ) is derived in the Appendix. This theory is similar to $N_{f}=4\left(\beta_{\mathrm{D}}=1\right)$, considered in this section, in that it also has diquarks with non-zero isospin: $u u$ and $d d$. The phase diagrams of these two theories are similar. 


\section{$7 \quad$ Large $\mu$ and FFLO}

The phase diagram we obtained using chiral perturbation theory is valid only as long as the chemical potentials remain small compared to the chiral scale, or $m_{\rho}$ which is the mass of the lightest hadron not included in the chiral Lagrangian. We can, however, study an opposite regime of very large $\mu$, when quarks are asymptotically free.

Let us first consider $N_{f}=2$. On the $\mu_{B}$ axis, $\mu_{I}=0$, the system is a Fermi liquid of $u$ and $d$ quarks with equal chemical potentials. The ground state is a superfluid state with nonzero $\langle u d\rangle$ condensate, which has the same quantum number as the diquark condensate considered previously in the framework of the chiral perturbation theory. Thus, it is natural to assume no phase transition on the $\mu_{B}$ axis. Analogously, the ground state at large $\mu_{I}$ contains $u \bar{d}$ Cooper pairs, since $u$ and $\bar{d}$ have the same Fermi energy; and there is likely no phase transition separating this phase from the low- $\mu_{I}$ phase of pion condensate. In both cases of large $\mu_{B}$ and $\mu_{I}$ all fermions acquire a gap $\Delta \sim \mu g^{-5} e^{-c / g}$ 14], here $c=3 \pi^{2} / \sqrt{2}$ and $g$ is the (small) gauge coupling. The proportionality factor has been calculated in 15$]$.

When $\mu_{B}$ and $\mu_{I}$ are nonvanishing, the chemical potential for the $u$ and $d$ quarks have different magnitudes. Generically, $\langle u d\rangle$ and $\langle u \bar{d}\rangle$ condensates cannot be formed unless the mismatch of the Fermi momenta is small. Thus, except for small regions near the $\mu_{B}$ and $\mu_{I}$ axes, in most of the $\left(\mu_{B}, \mu_{I}\right)$ plane the only condensates that can be formed are $\langle u u\rangle$ and $\langle d d\rangle$ (or $\langle\bar{d} \bar{d}\rangle$.) These Cooper pairs are color antisymmetric and flavor symmetric, and must hence carry spin or orbital moment and break rotational symmetry.

The region near the diagonal $\mu_{B}=\mu_{I}$ requires special consideration. On the diagonal $\mu_{d}=0$ and no $d$-quarks are present, while the $u$ quarks are paired. Below the scale of the BCS gap for the $u$ quark one should expect the $u$ quark to decouple completely. The $(E \ll \Delta)$ physics on the diagonal is thus $N_{f}=1 \mathrm{QCD}$ at zero chemical potential. The theory is confining [16] and one expects a gap for the lightest $d d$ baryon. This baryon cannot be generated unless $\mu_{d}$ is larger than this mass gap. Therefore, there is a strip along the diagonal where no $\langle d d\rangle$ or $\langle\bar{d} \bar{d}\rangle$ condensates are present.

Consider now the regions near the axes, say, the $\mu_{B}$ one. When $\mu_{I}$ is small no isospin charge is generated. One expects two phase transitions [11, 10], at $\mu_{I} \approx 0.71 \Delta$ and $0.75 \Delta$. In the narrow window the ground state is the FFLO phase with spatial varying diquark condensate. Similarly, there is a strip of another FFLO phase near the $\mu_{I}$ axis. We note that the evaluation in [10] applies equally well to $N_{c}=3$ and $N_{c}=2$ and support the occurrence of FFLO in two-colour two-flavour QCD.

We summarize what is said above in Fig. 5. Since we can solve our model analytically only in the two extreme limits, we have no information about how the lines are connected in the region of intermediate chemical potentials. Unfortunately, two colour QCD has a sign problem at $N_{f}=2$, so it is not clear whether one can find out about it on the lattice.

For $N_{f}=4$, it is more convenient to work in the variables $\mu_{u}$ and $\mu_{d}$. When $\left|\mu_{u}\right| \neq\left|\mu_{d}\right|$, the preferable pairing is $u u$ and $d d$ or $\bar{d} \bar{d}$. When $\mu_{d}=0$, the $(E \ll \Delta)$ theory reduces to vacuum QCD with $N_{f}=2$ which has confinement and a mass gap for the $d_{1} d_{2}$ diquark. When $\mu_{d}$ is less than $m_{d d}$, there should not be any $d$ quarks in the system. Thus, the phase diagram should resemble fig. 5 , where all lines are second order. The $u_{1} u_{2}$ condensate is always favourable and no FFLO-type transitions occur.

An interesting point is that the non-homogeneous FFLO phase can occur only in the $N_{f}=2$ case, and is not expected to arise in the $N_{f}=4$ case. This observation is a special case of a more general relation: the FFLO phase in Euclidean QCD is excluded if the measure is positive. This might be argued along the following lines, using QCD inequalities [17]. The measure in the QCD-like theories is real because of the additional symmetries of the Dirac operator, $D$. For $N_{c}=2\left(\beta_{\mathrm{D}}=1\right)$ at finite 


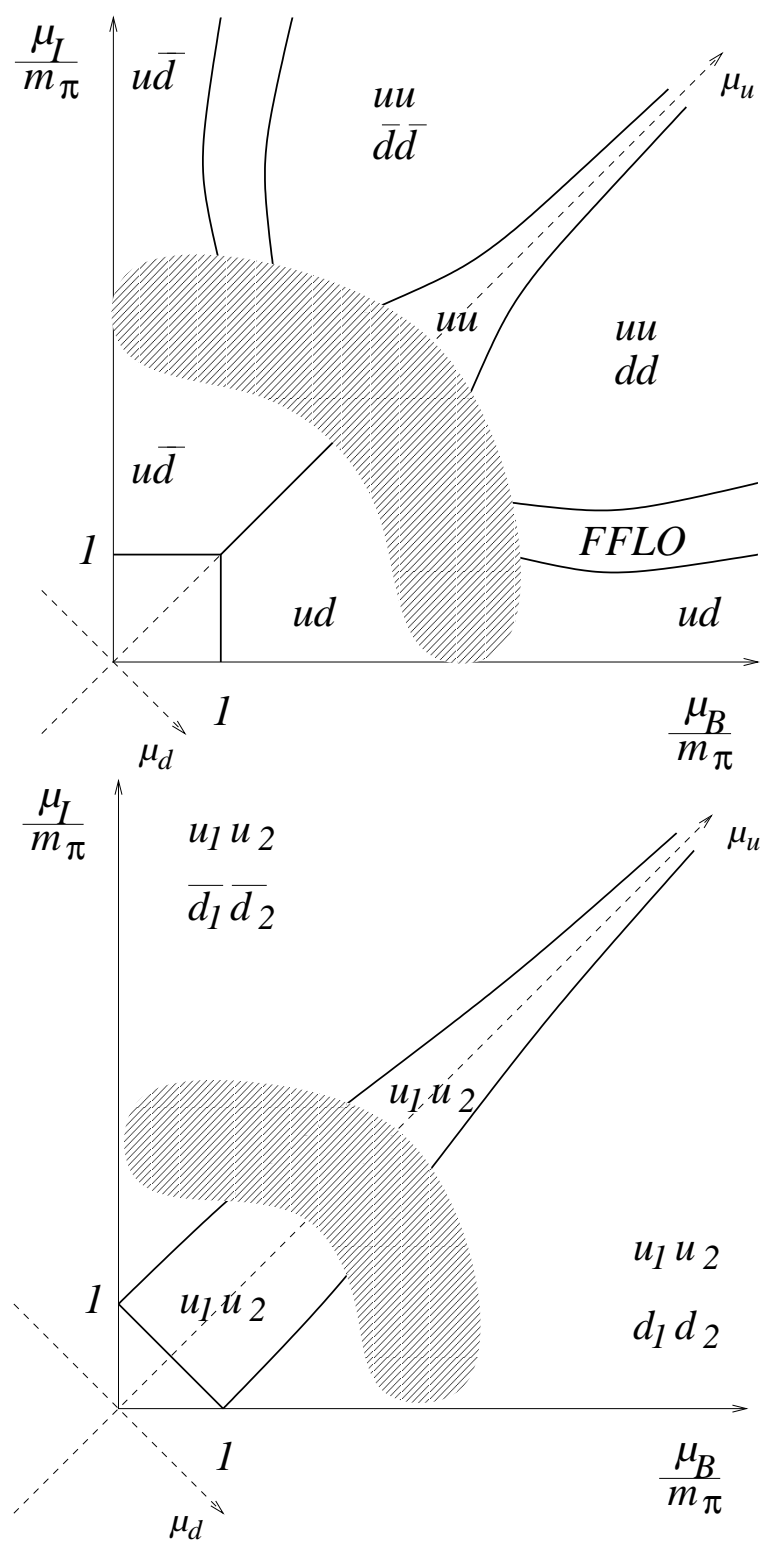

Figure 5: Two colour QCD at small and large chemical potentials: Upper figure shows $N_{f}=2$ and lower figure displays $N_{f}=4$. Solid lines are phase transitions and gray areas illustrate regions of phase space which remain undetermined. The lines above the clouds are drawn out of scale. Dashed lines show the direction of $\mu_{u}$ and $\mu_{d}$ axes. 
baryon and isospin chemical potential the additional symmetry is

$$
\sigma_{d} C \gamma_{5} \tau_{2} D \sigma_{d} C \gamma_{5} \tau_{2}=D^{*} \quad \text { with } \quad\left[\sigma_{d}, \sigma_{3}\right]=0 \quad \text { and } \quad \sigma_{d}^{2}=1
$$

where $C=i \gamma_{0} \gamma_{2}, \tau_{2}$ acts in colour space, and $\sigma_{3}$ in flavour space. This relation holds for $\mu_{B} \neq 0$ and $\mu_{I} \neq 0$. If the measure is positive (by doubling of flavours) the relation leads to a QCD-inequality for the diquarks [4. Since $\sigma_{d} C \gamma_{5} \tau_{2}$ only mix flavours with identical chemical potential so does the favored diquark channels for $\mu_{B} \neq 0$ and $\mu_{I} \neq 0$. However, a necessary condition for the FFLO phase is that the $u d$ diquark channel is dominant for $\mu_{B} \neq 0$ and $\mu_{I} \neq 0$ (for degenerate masses). Hence positivity excludes the FFLO phase. (The argument for QCD with quarks in the adjoint representation, $\beta_{\mathrm{D}}=4$, is equivalent - except in this case we need to assume that quark annihilation diagrams can be neglected.) It suggests that a relationship might exist between the presence of a translation-invariance-breaking phase and the (absence of) positivity in the corresponding Euclidean theory.

\section{Discussion and Conclusions}

One of the main obstacles to the study of QCD at finite baryon chemical potential is the loss of positivity of Euclidean path integral measure. Without the positivity standard lattice approach fails. One of the ways to gain insight into behavior of QCD at finite density is to study QCD-like theories with pseudo-real fermion content. Classified according to the Dyson index of the Dirac operator, $\beta_{\mathrm{D}}$, such theories are: $N_{c}=2 \mathrm{QCD}$ with fundamental quarks $-\beta_{\mathrm{D}}=1$, and any- $N_{c}$ QCD with adjoint quarks $-\beta_{\mathrm{D}}=4$.

Such QCD-like theories are studied here and in 4 , 5, 8]. We have studied the effects of simultaneous baryon and isospin chemical potentials in two limits: low and high density. The low energy limit is studied by means of effective low-energy approach - the chiral Lagrangian. Recent first-principle lattice studies of two-colour QCD at finite $\mu_{B}$ [6] have confirmed predictions from the effective theory at nonzero $\mu_{B}$, with $\mu_{I}=0$. The results of our paper are also testable. For $\beta_{\mathrm{D}}=1, N_{f}=4$ and $\beta_{\mathrm{D}}=4, N_{f}=2$ the fermion determinant is positive for all $\mu_{B}$ and $\mu_{I}$.

The low energy effective theory approach, in principle, applies even if positivity of the microscopic theory is lost. E.g., it applies to three colour QCD $\left(\beta_{\mathrm{D}}=2\right)$ at non-zero baryon and isospin chemical potential. However, the low-energy effective theory is valid only for energies much less than the smallest non-Goldstone hadron mass, and in this regime there is no effect of the baryon chemical potential. Low energy effective QCD is, on the contrary, affected if different chemical potentials are introduced for different quark flavours [18, 8]. The isospin case, where two quark chemical potentials of the same magnitude, but opposite sign, are introduced, was studied in [8]. Comparing that work with the one presented here we learn that $\mathrm{QCD}$ with $\beta_{\mathrm{D}}=1,2$, and 4 are very similar on the $\mu_{I}$-axis. In all cases a pion condensate forms at $\mu_{I}=m_{\pi}$.

In the $\mu_{B}, \mu_{I}$-plane the phase diagram distinguishes $\beta_{\mathrm{D}}=1,2$, and 4 . For $\beta_{\mathrm{D}}=1$ the pion condensate competes against the diquark condensate, and a novel first order phase transition takes place at $\mu_{B}=\mu_{I}>m_{\pi}$; for $\beta_{\mathrm{D}}=2$ there is no $\mu_{B}$ dependence (for $\mu_{B}<m_{N}$ ); and for $\beta_{\mathrm{D}}=4$ the $\mu_{u}$ and $\mu_{d}$ dependence separate (to lowest order in chiral perturbation theory).

In the high-density limit we have used weak coupling perturbation theory to study the phase diagram. We have found that theories with positivity do not possess a phase of inhomogeneous condensation - the FFLO phase. For example, $N_{f}=2 \mathrm{QCD}$ with adjoint quarks $\left(\beta_{\mathrm{D}}=4\right)$ is positive and does not display FFLO phase. On the contrary, $N_{f}=2$, 2-color QCD $\left(\beta_{\mathrm{D}}=1\right)$, does have regions of FFLO phase at large $\mu_{B}$ and $\mu_{I}\left(\mu_{B} \gg \mu_{I}\right.$ or $\left.\mu_{I} \gg \mu_{B}\right)$, while there is no positivity. 


\section{ACKNOWLEDGEMENTS:}

The authors thank the DOE Institute for Nuclear Theory at the University of Washington for its hospitality. KS thanks D. Toublan and J. Verbaarschot for discussions.

\section{A Quarks in the adjoint representation}

In this appendix we give results for QCD with quarks in the adjoint colour representation. This version of QCD does not have a problem with positivity. For $N_{f}=1$ the determinant is real and positive definite (see, e.g., the first reference of [6]). QCD with quarks in the adjoint colour representation belongs to the universality class labelled by Dyson index $\beta_{\mathrm{D}}=4$ [19], and its low energy limit is also described by chiral perturbation theory. Following the line of section 2 to 1 we consider $N_{f}=2$ and determine the phase diagram with $m \equiv m_{u}=m_{d}$. We expect to find a phase diagram similar to that of $\beta_{\mathrm{D}}=1, N_{f}=4$ since in both cases the lightest excitation on the $\mu_{B}$-axis is a diquark.

The symmetries of QCD with quarks in the adjoint colour representation allow for a chiral goldstone manifold with $N_{f}\left(2 N_{f}+1\right)-1$ degrees of freedom. The goldstone field $\Sigma$ may in this case be represented by a special unitary symmetric matrix [13]. The effective Lagrangian remains to be given by (3) provided that we replace $\mathcal{M}$ by

$$
\mathcal{M} \equiv\left(\begin{array}{cccc}
0 & 0 & 1 & 0 \\
0 & 0 & 0 & 1 \\
1 & 0 & 0 & 0 \\
0 & 1 & 0 & 0
\end{array}\right)
$$

The additional degrees of freedom as compared to $\left.\beta_{\mathrm{D}}=1\left(N_{f}\left(2 N_{f}+1\right)-1>N_{f}\left(2 N_{f}-1\right)-1\right)\right)$ allow for a different vacuum structure. The ansatz for the vacuum field is now

$$
\bar{\Sigma} \equiv \Sigma_{M}^{(u)} \cos \alpha_{u}+\Sigma_{u} \sin \alpha_{u}+\Sigma_{M}^{(d)} \cos \alpha_{d}+\Sigma_{d} \sin \alpha_{d},
$$

where $\Sigma_{M}^{(u)}, \Sigma_{M}^{(d)}, \Sigma_{u}$, and $\Sigma_{d}$ are defined as

$\Sigma_{M}^{(u)}=\left(\begin{array}{cccc}0 & 0 & 1 & 0 \\ 0 & 0 & 0 & 0 \\ 1 & 0 & 0 & 0 \\ 0 & 0 & 0 & 0\end{array}\right), \Sigma_{M}^{(d)}=\left(\begin{array}{cccc}0 & 0 & 0 & 0 \\ 0 & 0 & 0 & 1 \\ 0 & 0 & 0 & 0 \\ 0 & 1 & 0 & 0\end{array}\right), \Sigma_{u}=\left(\begin{array}{cccc}i & 0 & 0 & 0 \\ 0 & 0 & 0 & 0 \\ 0 & 0 & i & 0 \\ 0 & 0 & 0 & 0\end{array}\right), \Sigma_{d}=\left(\begin{array}{cccc}0 & 0 & 0 & 0 \\ 0 & i & 0 & 0 \\ 0 & 0 & 0 & 0 \\ 0 & 0 & 0 & i\end{array}\right)$.

With this ansatz the $\mu_{u}$ and $\mu_{d}$ terms do not mix at the classical level as can be seen from the Lagrangian at the minimum

$$
\mathcal{L}_{\text {eff }}(\bar{\Sigma})=-\frac{1}{2} F^{2}\left(-\cos \left(2 \alpha_{u}\right) \mu_{u}^{2}-\cos \left(2 \alpha_{d}\right) \mu_{d}^{2}+\mu_{u}^{2}+\mu_{d}^{2}+4 m_{\pi}^{2}\left(\cos \alpha_{u}+\cos \alpha_{d}\right)\right) .
$$

Extremizing with respect to the $\alpha$-directions we find:

$$
\begin{aligned}
\alpha_{f}=0 & \text { if } \quad \frac{m_{\pi}^{2}}{\mu_{f}^{2}}>1, \quad f=u, d \\
\cos \left(\alpha_{f}\right)=\frac{m_{\pi}^{2}}{\mu_{f}^{2}} \quad \text { if } \quad \frac{m_{\pi}^{2}}{\mu_{f}^{2}}<1 . &
\end{aligned}
$$


As for $\beta_{\mathrm{D}}=1$ we can prove that $\bar{\Sigma}$ is a local minimum by expanding around the minimum. The proof goes along the lines of the $\beta_{\mathrm{D}}=1$. First we choose a representation of the $\Pi$ field corresponding to the direction $\bar{\Sigma}=\mathcal{M}$. Instead of changing this representation as $\bar{\Sigma}$ rotate with increasing $\mu_{B}$ and $\mu_{I}$ we again choose to absorb the rotation in $\mu_{B} B+\mu_{I} I$ and $\mathcal{M}$. The analogue of Eq. (21) is

$$
\begin{aligned}
V^{\dagger}\left(\alpha_{u}, \alpha_{d}\right)\left(\mu_{B} B+\mu_{I} I\right) V\left(\alpha_{u}, \alpha_{d}\right)= & \left(\mu_{B} B+\mu_{I} I\right)\left[\left(\Sigma_{M}^{(u)}\right)^{2} \cos \alpha_{u}+\Sigma_{u} \Sigma_{M}^{(u) \dagger} \sin \alpha_{u}\right] \\
& +\left(\mu_{B} B+\mu_{I} I\right)\left[\left(\Sigma_{M}^{(d)}\right)^{2} \cos \alpha_{d}+\Sigma_{d} \Sigma_{M}^{(d) \dagger} \sin \alpha_{d}\right] \\
V^{T}\left(\alpha_{u}, \alpha_{d}\right) \mathcal{M} V\left(\alpha_{u}, \alpha_{d}\right)= & \Sigma_{M}^{(u)} \cos \alpha_{u}+\Sigma_{u} \sin \alpha_{u}+\Sigma_{M}^{(d)} \cos \alpha_{d}+\Sigma_{d} \sin \alpha_{d} .
\end{aligned}
$$

with

$$
V\left(\alpha_{u}, \alpha_{d}\right)=-i \Sigma_{u} e^{i \frac{\alpha_{u}}{2} \Sigma_{M}^{(u)}}-i \Sigma_{u} e^{i \frac{\alpha_{d}}{2} \Sigma_{M}^{(d)}} .
$$

Inserting these rotated versions of $\mu_{B} B+\mu_{I} I$ and $\mathcal{M}$ into $\mathcal{L}_{\text {eff }}(\bar{\Sigma})$ in Eq. (3) we get the $\beta_{\mathrm{D}}=4$ effective Lagrangian at $\left(\alpha_{u}, \alpha_{d}\right)$. Expanding this to second order in $\Pi$ one finds that the linear terms in $\Pi$ drop out due to the extremum conditions of $\mathcal{L}_{\text {eff }}(\bar{\Sigma})$ in Eq. (雨) with respect to $\alpha_{u}$ and $\alpha_{d}$. Hence the ansatz put forward in Eq. (2) is indeed a local extremum. Note that the quadratic terms in $\Pi$ mix the $u$ and $d$ sectors, i.e., the $u$ and $d$ sectors only separate at the classical level. As for $\beta_{\mathrm{D}}=1$ we will evaluate the condensates and densities classically. Assuming that the local extremum is a global minimum we draw the following conclusions

$$
\begin{aligned}
\langle\bar{\psi} \psi\rangle_{f} & =-\frac{\partial \mathcal{L}_{\text {eff }}(\bar{\Sigma})}{\partial m_{f}}=2 G \cos \alpha_{f}, \quad f=u, d \\
n_{B} & =-\frac{\partial \mathcal{L}_{\text {eff }}(\bar{\Sigma})}{\partial \mu_{B}}=2 F^{2}\left[\left(\sin ^{2} \alpha_{u}+\sin ^{2} \alpha_{d}\right) \mu_{B}-\mu_{I} \sin \left(\alpha_{u}+\alpha_{d}\right) \sin \left(\alpha_{d}-\alpha_{u}\right)\right] \\
n_{I} & =-\frac{\partial \mathcal{L}_{\text {eff }}(\bar{\Sigma})}{\partial \mu_{I}}=2 F^{2}\left[\left(\sin ^{2} \alpha_{u}+\sin ^{2} \alpha_{d}\right) \mu_{I}-\mu_{B} \sin \left(\alpha_{u}+\alpha_{d}\right) \sin \left(\alpha_{d}-\alpha_{u}\right)\right] .
\end{aligned}
$$

For $\beta_{\mathrm{D}}=4$ and $N_{f}=2$ two of the three diquarks are flavour diagonal, $\psi_{f}^{T} C \gamma_{5} \psi_{f}$. Introducing diquark sources for the diagonal ones we find

$$
\langle\psi \psi\rangle_{f}=2 G \sin \alpha_{f}, \quad f=u, d .
$$

The flavour mixing diquark has zero vacuum expectation value outside the $\mu_{B}$ and $\mu_{I}$ axis. On the $\mu_{B}$ axis it is of course degenerate with the two flavour-diagonal diquark condensates. For illustrations see figures 6 and 7 . Finally, let us comment on the possibility of realizing the FFLO state at large $\mu_{B}$ and small $\delta \mu_{I}$ (or visa versa). The theory allows for two flavour-diagonal diquarks and one flavour-mixing diquark. The two flavour diagonal diquark condensates are only mildly affected by $\delta \mu_{I}$. The flavour mixing state exists only on the $\mu_{B}$ axis as it competes against the flavour diagonal condensates. The FFLO state is therefore not expected to occur. This is consistent with the general relation stated at the end of section 0 .

\section{References}

[1] B. Barrois, Nucl. Phys. B 129, 390 (1977); S. Frautschi, Proceedings of workshop on hadronic matter at extreme density, Erice 1978; B.C. Barrois, Non-perturbative effects in dense quark matter, PhD Thesis, Caltech, 1979; D. Balin and A. Love, Phys. Rept. 107 (1984) 325, and references therein.

[2] M. Alford, K. Rajagopal, and F. Wilczek, Phys. Lett. B 422 (1998) 247; R. Rapp, T. Schäfer, E. V. Shuryak, and M. Velkovsky, Phys. Rev. Lett. 81 (1998) 53. 

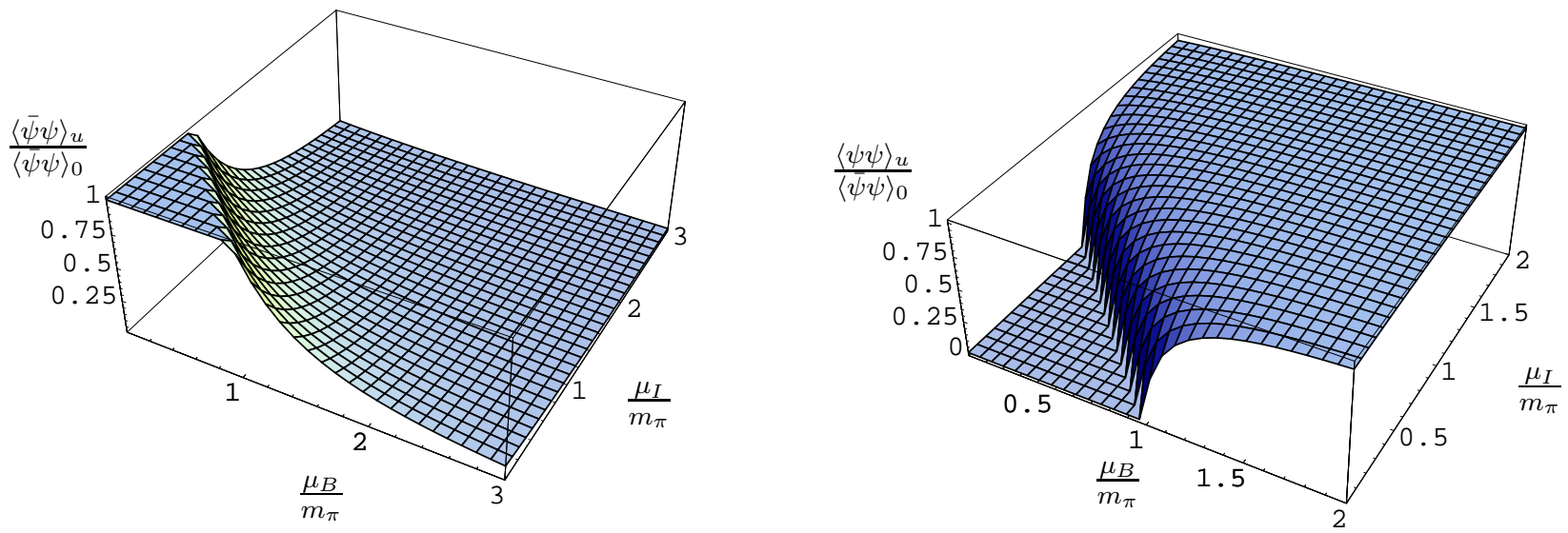

Figure 6: $\beta_{\mathrm{D}}=4$ : Left: Chiral condensate for $u$ in QCD with quarks in the adjoint colour representation. Measured in units of the chiral condensate at $\mu_{B}=\mu_{I}=0$. Right: Diquark condensate for $u$. Plots in the $d$-sector are obtained from these by a rotation about $\mu_{B}=\mu_{I}=0$ by $-\pi / 2$.
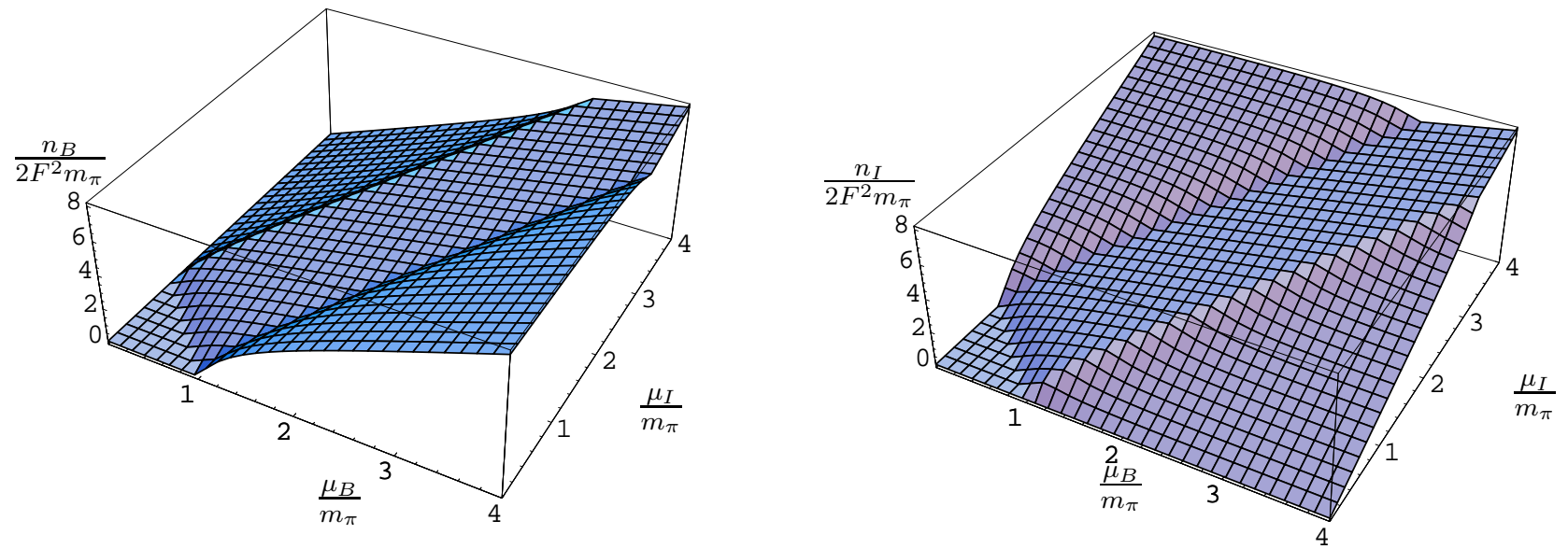

Figure 7: The baryon and isospin charge densities for $\beta_{\mathrm{D}}=4$. Left: $n_{B}\left(\mu_{B}, \mu_{I}\right)$. Right $n_{I}\left(\mu_{B}, \mu_{I}\right)$.

[3] K. Rajagopal and F. Wilczek, hep-ph/0011333.

[4] J.B. Kogut, M.A. Stephanov, and D. Toublan, Phys. Lett. B 464 (1999) 183-191.

[5] J.B. Kogut, M.A. Stephanov, D. Toublan, J.J.M. Verbaarschot, and A. Zhitnitsky, Nucl. Phys. B $\mathbf{5 8 2}(2000) 477-513$.

[6] S. Hands, I. Montvay, S. Morrison, M. Oevers, L. Scorzato, and J. Skullerud, Eur. Phys. J. C 17 (2000) 285-302;

R. Aloisio, V. Azcoiti, G. Di Carlo, A. Galante, A.F. Grillo, hep-lat/0007018 and Phys. Lett. B 493 (2000) 189-196;

Y. Liu, O. Miyamura, A. Nakamura, and T. Takaishi, hep-lat/0009009;

S.J. Hands, J.B. Kogut, S.E. Morrison, and D.K. Sinclair, hep-lat/0010028.

[7] M. Alford, A. Kapustin, and F. Wilczek, Phys. Rev. D 59, 054502 (1999).

[8] D.T. Son and M.A. Stephanov, hep-ph/0005225. 
[9] M.A. Stephanov, Phys. Rev. Lett. 76 (1996) 4472; Nucl. Phys. Proc. Suppl. 53 (1997) 469.

[10] M. Alford, J. Bowers, and K. Rajagopal, hep-ph/0008208.

[11] P. Fulde and A. Ferrell, Phys. Rev. 134 (1964) A550; A.I. Larkin and Yu.N. Ovchinnikov, Sov. Phys. JETP 20 (1965) 762.

[12] A. Smilga and J.J.M. Verbaarschot, Phys. Rev. D 51 (1995) 829.

[13] M. E. Peskin, Nucl. Phys. B 175 (1980) 197.

[14] D.T. Son, Phys. Rev. D 59 (1999) 094019.

[15] W. E. Brown, J. T. Liu, and H. Ren, Phys. Rev. D 62 (2000) 054016.

[16] D. H. Rischke, D. T. Son, and M. A. Stephanov, hep-ph/0011379.

[17] D. Weingarten, Phys. Rev. Lett. 51 (1983) 1830; S. Nussinov, Phys. Rev. Lett. 51 (1983) 2081; E. Witten, Phys. Rev. Lett. 51 (1983) 2351.

[18] D. Toublan and J.J.M. Verbaarschot, hep-th/0001110.

[19] J. Verbaarschot, Phys. Rev. Lett. 72 (1994) 2531-2533. 